\title{
Is logic in the mind or in the world?
}

\section{Gila Sher}

Received: 3 December 2009 / Accepted: 26 July 2010 / Published online: 9 October 2010

(C) The Author(s) 2010. This article is published with open access at Springerlink.com

\begin{abstract}
The paper presents an outline of a unified answer to five questions concerning logic: (1) Is logic in the mind or in the world? (2) Does logic need a foundation? What is the main obstacle to a foundation for logic? Can it be overcome? (3) How does logic work? What does logical form represent? Are logical constants referential? (4) Is there a criterion of logicality? (5) What is the relation between logic and mathematics?
\end{abstract}

Keywords Logic · Mind · World · Foundation · Logical constants · Criterion of logicality $\cdot$ Mathematics

My goal in this paper is to present an outline of a unified answer to the following questions:

1. Is logic in the mind or in the world?

2. Does logic need a foundation? What is the main obstacle to a foundation for logic? Can it be overcome?

3. How does logic work? What does logical form represent? Are logical constants referential?

4. Is there a criterion of logicality?

5. What is the relation between logic and mathematics?

I will address the first two questions individually, and offer an overall view of my answer to the last three.

This paper was presented at the Jean Nicod Institute (Paris), UC Santa Cruz, Pacific APA Symposium (Vancouver), and the Society of Exact Philosophy (Edmonton). I would like to thank the audiences, and in particular my commentators at the APA Symposium, Philip Hanson and Marcus Rossberg. Special thanks go to Clinton Tolley and Peter Sher.

G. Sher $(\bowtie)$

University of California, San Diego, 9500 Gilman Drive, La Jolla, CA 92093-0119, USA

e-mail: gsher@ucsd.edu 
Is logic in the mind or in the world?-Logic, I believe, like all other branches of knowledge, is grounded both in the mind and in the world, and its two grounds are interconnected. My answer is motivated both by general considerations pertaining to all branches of knowledge and by specific considerations pertaining to logic. The general considerations I will not discuss in detail here. The main point is that knowledge qua knowledge must be grounded both in its object, the world in a broad sense, and in its subject, the mind (also in a broad sense). Groundedness in the world is veridicality, i.e., compliance with strict standards of truth, evidence, and factual justification. Groundedness in the mind is conformity with pragmatic, conceptual, transcendental, linguistic, and possibly other extra-veridical norms.

Turning to special considerations, I will concentrate primarily on logic's grounding in the world, although I will say something about its grounding in the mind as well. The reason my study starts with the world is this: Throughout history, most systematic accounts of logic have focused on the mind and few on the world. As a result, many options for grounding logic in the mind are available to us, but only few for grounding it in reality. It follows that we are more likely to arrive at a unified grounding of logic in both if we attend to its grounding in the world first and use the result to constrain our account of its grounding in the mind (for which we have a rich trove of ideas to work with).

To prevent misunderstandings, let me say right away that by "world" and "reality" (which I use as synonyms) I mean neither "thing in itself" nor "mere appearances". Nor do I restrict reality to empirical experience or identify those aspects of the world that are relevant to logic with conceptual reality. Neither the Kantian duality of noumena and phenomena, nor pure Platonism or extreme empiricism are compatible with the present outlook. Furthermore, my claim that logic is both in the mind and in the world is not deflationist. Logic is both in the mind and in the world in a substantive sense, a sense that yields significant explanations, solves significant problems, and has significant consequences.

The view that logic is grounded in reality goes in the face of a powerful philosophical tradition: Kant regarded logic as grounded exclusively in the mind, as did the post-Kantian idealists; Carnap regarded logic as grounded in pragmatic conventions; and many contemporary philosophers regard logic as requiring no grounding at all. So, does logic require a grounding in reality? Does it require a grounding at all? And what does logic have to do with reality in any case? I will limit myself to five points:

(i) Logic's Special Standing in Our System of Knowledge. Logic has a special standing in our system of knowledge, both due to its great basicness and generality, and due to its normative force. Compare logic with physics, for example. Physics is bound by the laws of logic, but logic is (at least for the most part) not bound by the laws of physics; logic is conceivable outside of physics, but physics is inconceivable outside of logic; logic abstracts from the content of physical terms, but physics does not abstract from the content of logical terms; logic provides tools for physical theories, but physics (for the most part) does not provide tools for logical theories; a serious error in logic is likely to undermine physics, but (most) serious errors in physics are unlikely to undermine logic; and so on. Logic delineates some of the most basic forms of human thought and its expression, provides us with the most basic tools of valid inference, 
tells us what combinations of statements are permissible and impermissible, etc. Logical form, logical inference, logical criteria of consistency, are all ingredients that no system of knowledge can do without. Our system of knowledge can survive the removal of many a science, but not of logic. As a result, a grounding or a foundation for logic is crucial for our system.

(ii) The Importance of a Powerful wholesale Method of Inference. We have much to gain by having a well-founded logical system and much to lose without one. Due to our biological, psychological, intellectual, and other limitations, we, as agents of knowledge, can establish no more than a small part of our knowledge directly (or even relatively directly). Most items of knowledge have to be established through inference, or at least with considerable help of inference. A powerful method of inference is therefore indispensable for a system of knowledge built by and for humans. In particular, a powerful wholesale method - a wholesale method that enables us to increase the amount of our knowledge without reduction of truth, evidence, justification, or modal force-is of utmost importance. Not all methods of inference, however, are of this kind. Some powerful methods are narrow in scope, and some general methods are exceedingly weak. "Chemical inference", for example, is powerful but narrow in scope. If we know that water is $\mathrm{H}_{2} \mathrm{O}$, we can use this to expand our knowledge of phenomena involving water. But chemical inference is limited to physical knowledge, and indeed to a small portion thereof. Other types of inference are broad in scope but lacking in power. Material inference-mere preservation of truth-is a paradigm of such a method. Material consequences are found in all areas of knowledge, but they yield weak consequences, and therefore play no role in expanding our knowledge. Logical inference, in contrast, is both broad in scope and modally powerful. It enables us to draw inferences in all areas of knowledge, and its inferences preserve not just truth, but also evidence and modal status. As a result, logic is of immense value for our system of knowledge. Indeed, not only does logic enable us to extend our knowledge without reducing its veridicality or modal force, it enables us to reduce error by preventing inconsistencies, contradictions, and invalid inferences. All these strongly suggest that logical theory requires a grounding. We have too much to gain by a well-functioning logic, and too much to lose by an ill-functioning one, to afford an unfounded logic.

(iii) Logic Has to "Work" in the World. It is a simple and straightforward observation that logical theory, like physical theory, is correct or incorrect in the sense that it either "works" or "does not work" in the world. In the same way that the use of, say, defective aerodynamical principles can cause an airplane to malfunction, so the use of defective logical principles can result in its malfunctioning. If in designing an airplane we rely on incorrect logical laws-e.g., the law of "affirming the consequent", or the "new Leibniz law" (see (v) below) — we are likely to cause drag when lift is needed, a right turn when a left is intended, etc. A flawed logic can cause havoc in an airplane no less than a flawed physics. This is not to say that we have no latitude in constructing our logical (or physical) theory, but there is a very real sense in which our logical theory (like our physical theory) either works or does not work in the world. A useful logical theory has to avoid conflict with the world, just like any other theory. Adopting an 
influential argument from the philosophy of science, we may say that it would be a complete mystery that logic worked in the world if it were not tuned to the world.

(iv) Logic is Connected to Reality Through Truth. One way in which logic is inherently linked to reality is through its connection to truth. Take logical consequence. Consequence relations in general are relations of transmission or preservation of truth. If $\Gamma$ a set of sentences and $\mathrm{S}$ is a sentence, then:

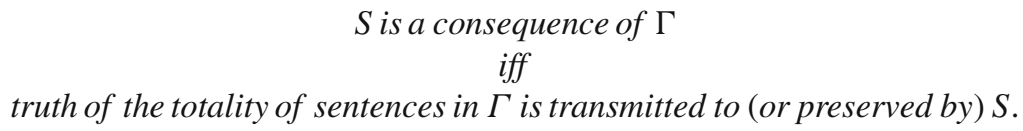

Truth, in general, inherently depends on whether things in the world are as given sentences say they are. Hence, whether $S$ is a logical consequence of $\Gamma$ inherently depends, in nontrivial cases, on whether the world being as the sentences in $\Gamma$ say it is guarantees (with a force sufficient for logical consequence) its being the way $\mathrm{S}$ says it is.

To see more clearly how judgments of logical consequence can be constrained by the world let us consider two sentences, $S_{1}$ and $S_{2}$, such that $S_{1}$ is true and the truth of both $S_{1}$ and $S_{2}$ is clearly a matter of whether certain things hold in the world. Let $\mathscr{L}$ be a logical theory saying that $\mathrm{S}_{2}$ is a logical consequence of $\mathrm{S}_{1}$. In symbols:

(2) (Level of Consequence) $\quad \mathrm{S}_{1} \mid={ }_{\mathrm{L}} \mathrm{S}_{2}$.

Then, if $\mathscr{L}$ is right, the truth of $\mathrm{S}_{1}$ guarantees the truth of $\mathrm{S}_{2}$. Figuratively:

(3) (Level of Truth) $\quad \mathrm{T}\left(\mathrm{S}_{1}\right) \rightarrow \rightarrow \rightarrow \mathrm{T}\left(\mathrm{S}_{2}\right)$.

Now, let $\mathfrak{S}_{1}$ and $\mathfrak{S}_{2}$ be the situations, or features of the world, on which the truth of $\mathrm{S}_{1}$ and $\mathrm{S}_{2}$ depends:

$\begin{array}{lcc}\text { (Level of Truth) } & \mathrm{T}\left(\mathrm{S}_{1}\right), & \mathrm{T}\left(\mathrm{S}_{2}\right) \\ & \mathbb{1} & \mathbb{1} \\ \text { (Level of World) } & \mathfrak{S}_{1} & \mathfrak{S}_{2} .\end{array}$

And suppose that in the world $\mathfrak{S}_{1}$ rules out $\mathfrak{S}_{2}$. Then, clearly, the truth of $\mathfrak{S}_{1}$ does not guarantee the truth of $S_{2}$, and $S_{2}$ is not a logical consequence of $S_{1}$. No matter what our logical theory says, the truth of $S_{1}$ does not guarantee that of $S_{2}$. Now suppose that $\mathfrak{S}_{1}$ does not rule out $\mathfrak{S}_{2}$ but simply $\mathfrak{S}_{1}$ is the case while $\mathfrak{S}_{2}$ is not the case. Then again $\mathrm{S}_{2}$ is not a logical consequence of $\mathrm{S}_{1}$. Finally, suppose that $\mathfrak{S}_{1}$ and $\mathfrak{S}_{2}$ are both the case, but $\mathfrak{S}_{1}$ does not necessitate (strongly guarantee) $\mathfrak{S}_{2}$. Again, $\mathfrak{S}_{2}$ is not a logical consequence of $\mathrm{S}_{1}$. In all these cases our logical theory, $\mathscr{L}$, is factually wrong: the truth of $\mathrm{S}_{1}$ does not guarantee the truth of $\mathrm{S}_{2}$. Figuratively:

\section{(Level of Consequence)}

(Level of Truth)

(Level of World)
NOT: $S_{1} \mid={ }_{L} S_{2}$

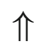

NOT: $\mathrm{T}\left(\mathrm{S}_{1}\right) \rightarrow \rightarrow \rightarrow \mathrm{T}\left(\mathrm{S}_{2}\right)$

介

$\mathfrak{S}_{1}=\Rightarrow \mathfrak{\Phi}_{2}$ or $\left\langle\mathfrak{S}_{1}, \mathbb{\Phi}_{2}\right\rangle$ or $\mathfrak{S}_{1}=\backslash \mathfrak{S}_{2}$. 
Of course, all this does not rule out the possibility that there is some built-in feature in judgments of logical consequence that protects them from ever being challenged by the world. But in that case we would have to veridically justify the claim that our logical theory is not falsified by the world. i.e., we have to ground logic in the world at least in a negative sense.

There are, however, reasons to believe that logic is grounded in the world also in a positive sense. i.e., some things in the world are significantly (if partially) responsible for the transmission of truth, or its failure, by putative logical consequences. This is because the world itself is capable of giving rise to consequences of various kinds, including logical. To see how this comes about, consider two situations in the world, $\mathfrak{S}_{1}$ and $\mathfrak{S}_{2}$, that correspond to two sentences, $\mathfrak{S}_{1}$ and $\mathfrak{S}_{2}$, respectively, as in (4) above. (Surely, there are such situations!) This case suffices to establish that $S_{2}$ is a material consequence of $S_{1}$. Figuratively:

(Level of Consequence Theory)
(Level of Truth)
(Level of World)

$$
\begin{gathered}
\mathrm{S}_{1} \mid={ }_{\mathrm{M}} \mathrm{S}_{2} \\
\mathrm{~T}\left(\mathrm{~S}_{1}\right) \rightarrow \underset{\Uparrow}{\rightarrow} \rightarrow \mathrm{T}\left(\mathrm{S}_{2}\right) \\
\\
\left\langle\mathfrak{S}_{1}, \mathfrak{S}_{2}\right\rangle .
\end{gathered}
$$

That is, the world can give rise to material consequences. Next, let us assume that $\mathfrak{S}_{1}$ nomically necessitates $\mathfrak{S}_{2}$, i.e., the world is governed by a law that positively connects $\mathfrak{S}_{1}$ and $\mathfrak{S}_{2}$ with some modal force (e.g., that of a physical law). Then, this will sanction not just a claim of material consequence, but a claim of nomic consequence. Figuratively:

$$
\begin{aligned}
& \text { (Level of Consequence Theory) } \\
& \text { (Level of Truth) } \\
& \text { (Level of World) }
\end{aligned}
$$$$
\begin{gathered}
\mathrm{S}_{1} \mid={ }_{\mathrm{N}} \mathrm{S}_{2} \\
\mathrm{~T}\left(\mathrm{~S}_{1}\right) \rightarrow \underset{\Uparrow}{\rightarrow} \rightarrow \mathrm{T}\left(\mathrm{S}_{2}\right) \\
\mathfrak{S}_{1} \Rightarrow \stackrel{\Uparrow}{\Rightarrow} \Rightarrow \mathfrak{S}_{2} .
\end{gathered}
$$

i.e., the world can give rise to nomic consequences.

Now, take any intuitive characterization of logical consequence in objectual terms or in terms that have objectual analogs. Say, "logical consequence is a universal, necessary, and formal consequence", where "formal" is spelled out in objectual terms. Suppose the law connecting $\mathfrak{S}_{1}$ and $\mathfrak{S}_{2}$ is universal, necessary, and formal (in the designated sense). Then, this law sanctions the claim that $S_{2}$ follows from $S_{1}$ with the force of a logical consequence. Figuratively:

$$
\begin{aligned}
& \text { (Level of Consequence) } \quad \mathrm{S}_{1} \mid={ }_{\mathrm{L}} \mathrm{S}_{2} \\
& \text { (Level of Truth) } \\
& \text { (Level of World) } \\
& \begin{aligned}
\mathrm{T}\left(\mathrm{S}_{1}\right) \rightarrow \underset{1}{\rightarrow} \rightarrow \mathrm{T}\left(\mathrm{S}_{2}\right) \\
\mathfrak{S}_{1} \rightarrow \stackrel{\Uparrow}{\rightarrow} \rightarrow \mathfrak{S}_{2} .
\end{aligned}
\end{aligned}
$$


For example, if the classical laws of meet and cardinality are necessary, universal, and formal, then the logical consequence " $(\exists x)(\mathrm{Px} \& \mathrm{Qx})$; therefore $(\exists \mathrm{x}) \mathrm{Px}$ " is sanctioned by the world:

(9) (Level of Consequence)

(Level of Truth)

(Level of World)

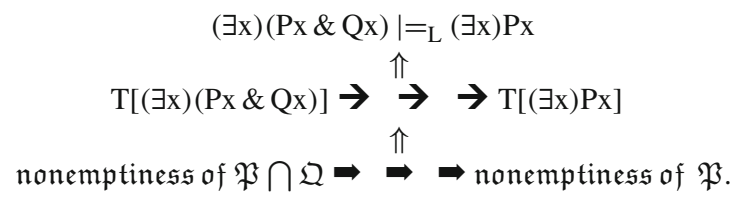

So the world can give rise to logical consequences. But if logic is empowered by the world, it is also constrained by it:

(10) (Level of Consequence)

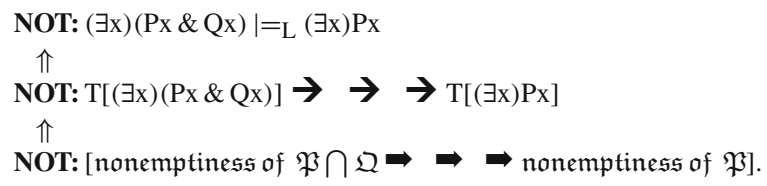

Logical consequence, then, is tied to reality through (i) its inherent connection with truth, (ii) the inherent connection between truth and reality, and (iii) the inherent relevance of (ii) to (i). ${ }^{1}$

These considerations suggest that logic is grounded in reality not in the traditional sense of being grounded in conceptual reality, but in the more robust and less mysterious sense of being grounded in certain laws governing the behavior of objects and properties in the world. i.e., logic is grounded in certain objectual features of reality. ${ }^{2}$

(v) The Threat of Factual Error in Logic. We have seen how, theoretically, logical judgments can be falsified, and justified, by the world. But the possibility of error in logic, including factual error, is not just a remote theoretical possibility. First, there are many advocates of nonclassical logics-free, fuzzy, intuitionistic, quantummechanical, paraconsistent, and others-who actually believe that "standard" (here, "classical") logic leads, at least potentially, to error or inefficacy.

Second, it is easy to construct "toy examples" that demonstrate the possibility of error, including factual error, in a logical theory. In a well known example Prior (1960) showed that an infelicitous choice of logical constants can introduce error into our system. Error, in logic, is typically inconsistency or contradiction, but a contradiction is, arguably, a factual error. Some contradiction-generating rules have a transparently factual dimension. Consider the following toy rule, which we can call "the new Leibniz Law": “ $\Phi(\mathrm{x}) ; \mathrm{x} \neq \mathrm{y} \vdash \sim \Phi(y)$ ". Whatever else is wrong with this rule, one thing that is wrong with it—and arguably responsible for its introducing a contradiction

\footnotetext{
1 I emphasize inherence to warn against drawing conclusions on this issue based on insignificant connections.

2 Note: this analysis can in a sense be read off the standard model theory of logic, but to treat it as derived from that theory is to put the cart in front of the horse. Rather, it is this analysis, or something like it, that explains why (something like) model-theoretic semantics displays, knowingly or unknowingly, a sound understanding of logic.
} 
into our system-is that it "says" that no (definable) property is common to multiple objects. And what about the non-contradiction-introducing rule "Water molecule (x) $\vdash \mathrm{H}_{3} \mathrm{O}$ molecule (x)". More than just violating certain requirements on logical constants, this rule is simply factually wrong. Of course, we can avoid these errors by a variety of restrictions on logical constants and rules of inference, but to determine what restrictions to introduce and to justify their appropriateness is just to provide a foundation for logic (or parts thereof).

The most compelling example of an error in logic, however, is an example of a devastating error in a "real life" logical theory, indeed one of the most distinguished theories in the history of logic: Frege's theory. Russell's paradox conclusively proved that there was a fatal error in Frege's logic, and it is widely believed that the source of this error is factual: Frege's logic is committed to the existence of an object-a class - that does not (and cannot) exist.

These are some of the considerations that lead me to think that logic requires a foundation, including a foundation in reality. But is a foundation for logic possible? Can we, in principle, provide logic with a substantive foundation, be it in the mind, in reality, or in both?

Traditionally, the greatest obstacle to a foundation for logic was thought to be circularity (infinite regress). The crux of the matter is the "'logocentric' predicament": "In order to give an account of logic, we must presuppose and employ logic" (Sheffer 1926, p. 228). In Wittgenstein's idiom: To provide a foundation for logic we have to stand "somewhere outside logic", but there is no cognitive standpoint outside logic (1921, 4.12).

Is the logocentric predicament unsurpassable? Does it rule out in advance the possibility of a foundation for logic? I believe it does not. This alleged predicament is a remnant of foundationalism - a widely discarded methodology that still affects philosophers' conception of a foundation for logic. And by consistently eschewing this methodology we avoid this predicament. Let me explain.

Foundationalism purports to provide a grounding for knowledge (in whatever a given foundationalist theory purports to ground it) in a simple and straightforward manner. Focusing on a foundation in reality, we can describe the foundationalist method succinctly as follows:

(i) Foundational items of knowledge are grounded in reality directly, through direct experience or intuition.

(ii) Derivative items of knowledge are grounded in reality indirectly, through reliable knowledge-extending procedures which are themselves either foundational or derivative.

A salient, and often overlooked, feature of foundationalism is its strict ordering requirement. Foundationalism imposes a strict ordering on our system of knowledge by requiring the grounding relation to (i) be (in paradigmatic cases) irreflexive, anti-symmetric and transitive, (ii) have an absolute base of minimal elements, and (iii) connect each nonminimal element to one or more minimal elements by a finite chain. This salient feature of foundationalism is both a source of its promise and a cause of its failure. On the one hand, it enables foundationalism to reduce the unmanageable task of providing a grounding for every item of knowledge to the more manageable 
task of providing a grounding only to the basic units. On the other hand, it leaves foundationalism with no resources for grounding the basic units.

Now, since, due to its special standing, logic is placed at the bottom of the foundationalist hierarchy, foundationalism is incapable of providing a foundation for logic. Placing logic at the base means that while logic can provide (partake in providing) a foundation for other sciences, no science(s) can provide a foundation for logic. Yet, since a serious error in logic will undermine our entire system of knowledge, a foundation for logic, more than for any other science, must be provided. Must and cannot. Having postulated (i) that any resource for founding logic must be more basic than those produced by logic itself, and (ii) that there are no resources more basic than those produced by logic, foundationalism has no resources for constructing a foundation for logic.

The key to a viable foundation for logic (and knowledge in general) is, I believe, separating the foundational project from the foundationalist methodology, and in particular rejecting the strong ordering requirement. My line of reasoning is the following: If we demand that the grounding relation be strongly ordered, we undermine the foundational project. But why should the grounding relation be required to have this formal feature? It is true that logic itself provides a model for a strongly ordered grounding relation, namely, the deductive method (in its most basic form), but this does not mean that the grounding of this method itself, let alone of all of knowledge, must follow the pattern exemplified by logic. Rather, the grounding relation may follow multiple formal patterns, some exemplifying strict orderings, others not. That is to say, the grounding relation may be strictly ordered in some places, have other features in others.

Relaxing the strong ordering requirement by itself, however, will not necessarily lead to a foundational methodology in our sense, namely, a methodology that mandates the grounding of knowledge in reality. A prime example of a methodology that does not require such a grounding is coherentism. Indeed, foundationalism and coherentism mark two opposites of the foundation divide. Using three parameters-(i) strong ordering of the grounding relation, (ii) use of knowledge-based resources in the grounding process, and (iii) grounding of knowledge in reality - we can display their opposition by the following table:

\begin{tabular}{llll}
\hline & $\begin{array}{l}\text { Strong ordering } \\
\text { of the } \\
\text { grounding } \\
\text { relation }\end{array}$ & $\begin{array}{l}\text { Use of knowledge- } \\
\text { based } \\
\text { resources in the } \\
\text { grounding process }\end{array}$ & $\begin{array}{l}\text { Grounding } \\
\text { knowledge } \\
\text { in reality }\end{array}$ \\
\hline $\begin{array}{l}\text { Foundationalism } \\
\text { Coherentism }\end{array}$ & Required & Restricted & Required \\
\hline
\end{tabular}

Foundationalism and coherentism, however, do not exhaust all possibilities with respect to these parameters. In particular, one configuration left out is:

\begin{tabular}{llll}
\hline Strong ordering & $\begin{array}{c}\text { Use of knowledge } \\
\text { based resources }\end{array}$ & $\begin{array}{c}\text { Grounding knowledge } \\
\text { in reality }\end{array}$ \\
\hline Not required & Unrestricted & Required \\
\hline
\end{tabular}


I will call this configuration foundation without foundationalism (a variation on the title of Shapiro 1991). Foundation without foundationalism shares foundationalism's commitment to grounding all knowledge in reality while renouncing its rigid methods. It says that in grounding our system of knowledge in reality we need not encumber ourselves with unreasonable restrictions, and it grants us maximum freedom in carrying out the grounding project. Unlike foundationalism, it does not determine in advance either the formal structure of, or the resources used in, each stage of the grounding process; and unlike coherentism, its does not give up, or in any way compromise, our veridicality standards.

The foundation-without-foundationalism strategy is a holistic strategy. It is not holistic in the sense, rightly criticized by Dummett (1973), of treating our entire system of knowledge as a single, undifferentiated, whole; it is holistic in the sense of emphasizing the existence of a variegated, open-ended network of relations encompassing all units of knowledge. I will call a methodology advocating this strategy "foundational holism".

A characteristic metaphor of foundational holism is "Neurath's boat". To check the soundness of the boat we find a temporary foothold in a relatively sound area of the boat, use available resources to find and repair holes, and continue sailing. Once we have mended one area of the boat, we can use it as a temporary standpoint for checking and repairing other areas. We may create new resources for repatching (better patching) the original holes, and so on. In this way all sections of the boat can be repaired, and different sections can be used as foothold.

Neurath's-boat is often viewed as a coherentist metaphor. But it is just as much, and even more so, a metaphor of foundational holism. First, Neurath's boat, as representing our system of knowledge, does not hover in empty space, but travels in a real, external, resistant medium: sea or ocean. This medium exerts real pressures upon it (buffets it with real waves, etc.). And this means that its sailors must take reality into account. A hole in the boat cannot be patched with a (regular) scotch tape, and in steering the boat the sailors must harness the forces of nature while guarding against its dangers. Moreover: Not only is the boat not hovering in empty space, it is not aimlessly floating in the water either. Neurath's boat is not a pleasure boat or a dinghy adrift in sea, but a vessel on an expedition, headed in a special direction, and having a mission to accomplish. Its mission is to study the sea and its environs, and its strategy is to use any available resources, internal and external, in any possible way, strictly ordered or not. As such it is invested in the world, directed toward the world, and constrained by the world, though in a holistic rather than in a foundationalist manner.

Having identified foundational holism as our non-foundationalist method for grounding logic in reality, our task is to develop an actual grounding for logic using this method. I will pursue this task by starting with an analysis of logic's connection to reality. Then, exploiting the holistic license to employ resources developed by other disciplines, I will identify the features of reality that logic is grounded in and explain how it is grounded in these features. Since I have elaborated on some of the specific issues involved in this grounding elsewhere, I will focus on the overall conception here.

The central idea is that logic is grounded in formal or structural laws governing the world-laws governing the formal (structural) features of objects, or their formal 
behavior. This notion of a formal law is an objectual notion and it is closely related to the objectual conception of a mathematical law in the philosophy of mathematics. As a result, our solution forges a close link between logic and mathematics, although a link quite different from those forged by earlier philosophers, be they logicists, intuitionists, or (Hilbertian) formalists. ${ }^{3}$

One way to arrive at our solution is to start with the basic schema connecting logic to reality through truth:

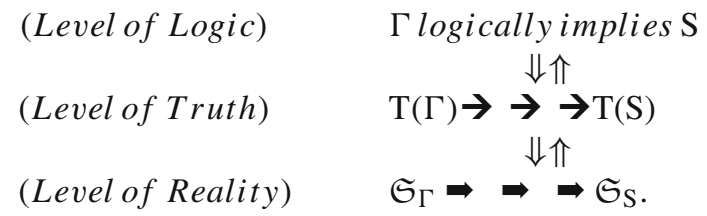

The upward arrows represent a strong-guarantee relation, and the downward arrows represent dependence. We know that $\Rightarrow \Rightarrow \vec{b}$ is a strong necessitation relation, so we can treat it as representing a law. Our question is: What type of law connecting objects and structures of objects in the world is sufficiently and appropriately strong to ground logical consequence? To answer this question we need to have some idea about the special strength of logical consequence. One way to go about this is to turn to the common attributes of logic.

The traits most commonly attributed to logic are necessity, great generality, formality, apriority, analyticity, topic neutrality, strong normative force, and certainty. Not all of these are compatible with our conclusion that logic is substantially (if not exclusively) grounded in reality. In particular, analyticity is incompatible with it. Thus, one significant result of our view is rejection of the claim that logic is (fully) analytic. Another attribute that stands in some tension with our approach is apriority, but here the situation is more nuanced. While traditional or absolute apriority is incompatible with our holistic approach to logic, the so-called new or relative a priori (see, e.g., Boghossian and Peacocke 2000) is compatible with it. In particular, the view that reason, as distinct from sensory experience, plays a central (if not an exclusive) role in logic is concordant with our approach. We are left, then, with the attributes of necessity, formality, relative apriority, topic neutrality, strong normative force, great generality, and certainty. ${ }^{4}$ Our question now becomes: Is there a type of (objectual) law that has all these attributes?

Examining the (objectual) laws characteristic of different sciences, we see that there is one type of law that does: mathematical law, and in particular "basic" mathematical law. A few examples of such a law are the laws of complementation, join, meet, identity, and finite cardinality. These laws are necessary, formal, relatively a priori, and

\footnotetext{
3 My objectual view of mathematical law is similar to that of contemporary structuralists, but in viewing mathematics as closely related to logic I am closer to the logicists, intuitionists, and formalists than to most structuralists. One structuralist who allows a close relation between logic and mathematics is Shapiro (1997), but even he prefers to leave this issue an open question.

4 In saying that logic is intuitively "certain" I do not mean to say that we cannot err in our logical inferences. Rather I mean to say that when we do not err, our logical inferences can be relied on without reservation, unlike, say, our inductive inferences.
} 
highly certain; they are also highly general and topic neutral (they have an especially wide range of applications); and their normative force is stronger than that of most other laws (in the sense that generally laws of other fields - say, biological laws- have to obey them, but not vice versa).

To show that this connection is not ad hoc, let us show how it develops from an independent characterization of the formal. Sharpening our intuitions by means of an example, let us ask: What formal law is the logical consequence

$$
(\exists \mathrm{x})(\mathrm{Px} \& \mathrm{Qx}) \mid={ }_{\mathrm{L}}(\exists \mathrm{x}) \mathrm{Px}
$$

grounded in? Our earlier discussion suggested the law:

(13) nonemptiness of $\mathfrak{P} \bigcap \mathfrak{Q} \Rightarrow \Rightarrow \Rightarrow$ nonemptiness of $\mathfrak{P}$,

where $\mathfrak{P}$ and $\mathfrak{Q}$ are any properties of individuals. Now consider (a correct statement about) the failure of a purported logical consequence:

(14) $(\exists \mathrm{x})(\mathrm{Px} \vee \mathrm{Qx}) \not \nvdash_{\mathrm{L}}(\exists \mathrm{x}) \mathrm{Px}$.

Where does the failure of this consequence lie?-A natural answer is: In the fact that

(15) NOT: [nonemptiness of $\mathfrak{P} \bigcup \mathfrak{Q} \Rightarrow \Rightarrow \Rightarrow$ nonemptiness of $\mathfrak{P}]$.

These examples suggest that formal laws are laws governing such features of objects as meet, join, and cardinality. How can we characterize these features?

Historically, one way in which philosophers have characterized the formality of logic is in terms of not distinguishing differences between, or particular features of, objects (Kant 1781/1787; Frege 1879), or being unaffected by 1-1 replacements of objects (Tarski 1936). To be more specific, we suggest the following criterion of formality (a generalization of Tarski 1966):

(F) To be formal is not to distinguish any replacement of objects (properties, relations, functions) induced by 1-1 replacements of individuals within and across any domains.

Using the resources of modern mathematics (which we are licensed to do by our holism), we can reformulate (F) as an Invariance Criterion:

(IF) To be formal is to be invariant under isomorphisms of structures,

where a structure is an $\mathrm{n}+1$-tuple, $\left\langle\mathrm{A}, \beta_{1}, \ldots, \beta_{\mathrm{n}}\right\rangle$, such that $\mathrm{A}$ is a nonempty set of objects viewed as individuals, and for $1 \leq \mathrm{i} \leq \mathrm{n}, \beta_{\mathrm{i}}$ is either a member/subset/relation/function of (on) A, or a set/relation/function of subsets/relations/functions/(plus, possibly, individuals) of A, etc.

To see how this criterion works in a particular case, take the feature of nonemptiness. This feature is (objectually) formal since for any (objectual) structures $\langle\mathrm{A}, \mathrm{B}\rangle$ and $\left\langle\mathrm{A}^{\prime}, \mathrm{B}^{\prime}\right\rangle$, where $\mathrm{A}$ and $\mathrm{A}^{\prime}$ are as above and $\mathrm{B}$ and $\mathrm{B}^{\prime}$ are any subsets of $\mathrm{A}$ and $\mathrm{A}^{\prime}$ respectively (representing possible arguments of " $\mathrm{X}$ is nonempty" in $\mathrm{A}$ and $\mathrm{A}^{\prime}$ ), the following holds:

(16) $\left[\operatorname{Nonempty}(\mathbf{B}) ;\langle\mathbf{A}, \mathbf{B}\rangle \cong\left\langle\mathbf{A}^{\prime}, \mathbf{B}^{\prime}\right\rangle\right] \Rightarrow \Rightarrow \operatorname{Nonempty}(\mathbf{B})$. 
What kind of law is responsible for logical consequences involving the formal property of nonemptiness? (E.g., What kind of law is (13)?) Well, a mathematical law that can be formulated by various theories within mathematics (e.g., ZFC Zermelo-Fraenkel set theory with the axiom of choice). It is easy to see that all logical consequences of classical logic are supported by laws of this kind and all failures of consequence according to this logic can be attributed to a violation of such laws. (In the case of logical consequences of classical sentential logic, formality is bivalence, and the relevant formal laws are formulated by a 2-element Boolean algebra. In the case of nonclassical logic, the formal laws are given by nonclassical mathematical theories.) This gives us a direct connection between logic and mathematics.

Our characterization of logic in terms of formality is further supported by the consideration that formality (in our sense) entails necessity, generality, topic-neutrality, and quasi-apriority, while none of these attributes entails formality. Take, for example, quasi-apriority. Due to their strong invariance, the formal properties, and hence the laws governing them, are indifferent to most variations between structures of objects, including all variations concerning empirical differences between structures. This, in turn, means that formal laws are not affected by most discoveries made in the sciences, hence are quasi-a priori. (Being holists, we do allow that some theoretical considerations concerning science may suggest a change in formal laws. But for the most part these laws are not affected by the vicissitudes of science.) Similar considerations show that formality entails necessity, generality, topic neutrality, and certainty (see Sher 1999, 2001, 2008). Therefore, by characterizing logic in terms of formality we capture many of its traditional attributes.

All these considerations suggest the following picture of the grounding of logic:

A. Logic provides a method of inference based on the formal laws governing the behavior of objects in the world.

B. This it does by taking parts of language that are dedicated to the formal, holding them "fixed", and integrating them into the syntactic structure of our language as logical constants. Logical constants, on this conception, denote formal properties (relations, functions) of objects. Logical form represents the formal structure of whatever given sentences claim to be the case. (For example, the logical form of “ $(\exists \mathrm{x})($ Planet $\mathrm{x} \&$ Frozen $\mathrm{x})$ " represents a nonempty intersection.)

C. There is a division of labor between logic and mathematics concerning the formal: The formal is studied in mathematics; employed as a basis for a wholesale method of inference in logic.

D. Logic and mathematics are developed in tandem. Starting with some basic formal principles, a basic mathematics is used to develop a logic which, in turn, is used to develop a more sophisticated mathematics. This mathematics is used to develop a still more sophisticated logic, and so on. Although our account is functional rather than historical, the mutually sustaining development of modern logic and set theory exemplifies our conception.

E. In developing logic using mathematical resources, several courses of action are open to us. At the two extremes we have opposing methodological principles: (i) Minimize, and (ii) Maximize. The former leads to the construction of minimal logics (e.g., standard 1st-order logic); the latter to the construction of 
maximal logics (e.g., generalized 1st-order logics and full 2nd-order logic). Taking the Minimize route (and limiting ourselves to classical logics), our criterion of formality-IF (plus the Boolean criterion for sentential-operators) - is understood as a constraint on logicality, i.e., as a necessary condition on the choice of logical operators. Taking the Maximize route, this criterion becomes a full criterion of logicality, i.e., a necessary and sufficient condition on the choice of logical operators. (A non-classical conception of the formal structure of reality will lead to appropriate adjustments in IF and the Boolean criterion and, consequently, to a choice of non-classical logical operators.)

Much work remains to be done in developing our conception of logic as grounded both in the mind and in reality. For a further elaboration of (A)-(E), as well as some of the other themes developed in this paper, see Sher (1991, 1996, 1999, 2001, 2002, 2003, 2008).

Open Access This article is distributed under the terms of the Creative Commons Attribution Noncommercial License which permits any noncommercial use, distribution, and reproduction in any medium, provided the original author(s) and source are credited.

\section{References}

Boghossian, P., \& Peacocke, C. (Eds.). (2000). New essays on the a priori. Oxford: Oxford University Press.

Dummett, M. (1973). Frege: Philosophy of language. New York: Harper \& Row.

Frege, G. (1879). Begriffsschrift: A formula language, modeled upon that of arithmetic, for pure thought. In J. van Heijenoort (Ed.), From Frege to Gödel (pp. 5-82). Cambridge: Harvard, 1967.

Kant, I. (1781/1787). Critique of pure reason (1st and 2nd ed., Trans. P. Guyer \& A. W. Wood). Cambridge: Cambridge University Press, 1998.

Prior, A. N. (1960). The runabout inference-ticket. Analysis, 21, 38-39.

Shapiro, S. (1991). Foundations without foundationalism: A case for second-order logic. Oxford: Oxford University Press.

Shapiro, S. (1997). Philosophy of mathematics: Structure \& ontology. New York: Oxford University Press.

Sheffer, H. M. (1926). Review of Principia mathematica by Whitehead, A.N.; Russell, B. Isis, 8, $226-231$.

Sher, G. (1991). The bounds of logic: A generalized viewpoint. Cambridge: MIT.

Sher, G. (1996). Did Tarski commit 'Tarski's fallacy'?. Journal of Symbolic Logic, 61, 653-686.

Sher, G. (1999). Is logic a theory of the obvious?. European Review of Philosophy, 4, 207-238.

Sher, G. (2001). The formal-structural view of logical consequence. Philosophical Review, 110, 241-261.

Sher, G. (2002). Logical consequence: An epistemic outlook. Monist, 85, 555-579.

Sher, G. (2003). A characterization of logical constants is possible. Theoria, 18(2003), 189-197.

Sher, G. (2008). Tarski's thesis. In D. Patterson (Ed.), New essays on Tarski and philosophy (pp. 300-339). Oxford: Oxford University Press.

Tarski, A. (1936). On the concept of logical consequence. In J. Corcoran (Ed.), Logic, semantics, metamathematics (J. H. Woodger, Trans., 2nd ed. 1983, pp. 409-420). Indianapolis: Hackett.

Tarski, A. (1966). What are logical notions? In J. Corcoran (Ed.), History and philosophy of logic 7 (1986), 143-154.

Wittgenstein, L. (1921). Tractatus logico-philosophicus (Pears \& McGuinness, Trans., 1961). London: Routledge \& Kegan Paul. 